Indonesian Journal of Medicine (2019), 4(1): 28-34

https://doi.org/10.26911/theijmed.2019.04.01.05

\title{
A High Incidence of Multidrug Resistant Strains as the Agents Causing Bloodstream Infection in Hospital in Indonesia
}

\author{
Betty Suryawati'), Leli Saptawati ${ }^{1,2)}$ \\ 1)Department of Microbiology, Faculty of Medicine, Universitas Sebelas Maret, \\ ${ }^{2)}$ Clinical Microbiology Laboratory, Dr. Moewardi Hospital, Surakarta
}

\begin{abstract}
Background: Various bacteria have been identified as the agents causing bloodstream infection. Multidrug resistant (MDR) strains, such as Gram-negative pathogens producing extended-spectrum beta-lactamases (ESBL) and Methicillin-resistant S.aureus (MRSA) are potential bacterial agents causing bloodstream infection in the hospital setting. The increase of these MDR strains in bloodstream infection is alarming since there are limited antibiotic choices for treatment. This study aimed to investigate the agents causing bloodstream infection and to investigate their antibiotics susceptibility profiles.

Subjects and Method: Samples were venous blood collected from patients admitted to hospital from January 2014 to December 2016. Bacteria were grown in-automated BACTEC@ blood culture (BD, New Jersey, USA). The identification of MRSA strain was conducted using oxacillin susceptibility test. The bacterial identification and identification of ESBL-producing bacteria was conducted using colorimetric assay using Vitek2®system (bioMérieux).

Results: The antibiotic profiles of all bacteria isolated from venous blood samples were analysed. The most bacteria isolated from the blood stream were $K$. pneumonia, A. baumannii, E. coli, $P$. Aeruginosa, E. cloacae, S. marcennes, S. haemolyticus, S. hominis, S.epidermidis, and $S$. aureus.

Conclusions: The resistant profiles of bacteria isolated from blood stream showed that there was a high incidence of MDR bacteria, including potential ESBL-producing bacteria, carbapenemaseproducing bacteria and MRSA strains.
\end{abstract}

Keywords: Bloodstream Infection, Staphylococcus aureus, Extended Spectrum Betalactamase, Antibiotic Resistance.

\section{Correspondence:}

Betty Suryawati. Department of Microbiology, Faculty of Medicine, Universitas Sebelas Maret, Jl. Ir. Sutami No 36A, Surakarta 57126, Central Java, Indonesia. Email: betty.suryawati@staff.uns.ac.id.

\section{BACKGOUND}

$\overline{\text { Bloodstream infection is a serious infection }}$ caused by the presence of bacteria in the bloodstream. Various bacteria have been identified in blood samples, including coagulase-negative staphylococci (CoNS), Staphylococcus aureus (S. aureus), and enterococci (Hoenigl et al., 2014, Lim et al., 2014). Recently, there is an increase of multidrug resistant (MDR) age strains causing bloodstream infection in hospital setting, such as Gram-negative pathogens producing extended-spectrum beta-lacta- mases (ESBL) and Methicillin-resistant $S$. aureus (MRSA) (Corcione et al., 2018, Kuntaman et al., 2016, Hasso et al., 2017, Pichler et al., 2018). MRSA is a strain of $S$. aureus that shows resistance to various antibiotics, such as ampicillin, penicillin, oxacillin, and other common antibiotics. ESBL-producing bacteria are Gram-negative bacteria that produce beta-lactamase enzyme. This enzyme causes the bacteria resistance to antibiotics, such as penicillin and cephalosporin and because their ability to break down antibiotics. Infection by 
ESBL-producing bacteria and MRSA are now common in hospital setting. The infection by these strains has increased the inadequate treatment and subsequently increased the mortality rate(RodriguezBano et al., 2008).

There are previous studies about blood stream infection and the agents commonly caused the infection in community and hospital setting reported worldwide (Hasso et al., 2017); however there is limited publication about the agent causing bloodstream infection and their resistant profiles in Surakarta, Indonesia. A regional antibiotic profile of agents is important to determine the empirical treatment since the resistant profile may differ between regions. A study in a hospital in Jakarta, Indonesia showed that Gram-negative bacteria were responsible to cause blood stream infection in neonatus, that were Enterobacter asburiae (E. asburiae), Enterobacter cloacae (E. cloacae), Pseudomonas aeruginosa ( $P$. aeruginosa) and Klebsiella oxytoca (K. oxytoca) (Moehario et al., 2012). Another study reported that MDR Acinetobacter baumannii (A. baumannii) was found in neonatal blood stream infection (Tjoa et al., 2013).

The aim of this study was to investigate the agents causing bloodstream infection and their resistant profilesin a teaching hospital, in Surakarta, Central java, Indonesia. The knowledge about agents commonly found in bloodstream and the resistant profile locally is very important to determine the effective therapy and prevention approach to reduce mortality rate due to blood stream infection.

\section{SUBJECTS AND METHOD}

\section{Study Design}

This was a retrospective descriptive study conducted in Dr. Moewardi Hospital, Surakarta, Central Java, Indonesia. The hospital is a public teaching hospital with more than 800 beds, affiliated to Faculty of Medicine, Universitas Sebelas Maret, Surakarta, Indonesia. The samples were all blood stream isolates obtained from patients presented with clinical manifestations of bloodstream infections from January 2014 to December 2016 collected at the Clinical Microbiology Laboratory of Dr. Moewardi Hospital.

\section{Specimen, culture, and identifica- tion}

Blood samples were collected from patients with clinical manifestations of bloodstream infections admitted to Moewardi Hospital from January 2014 to December 2016. Blood was cultured in BACTEC $\AA$ (BectonDickinson, New Jersey, USA) bottles. For children, BACTEC®Peds Plus/F bottles were used. When there was bacterial growth indicated by the BACTEC machine, blood was sub-cultured onto a MacConkey agar and blood agar plates. The colonies grown in the plates were used for Gram staining and antibiotic susceptibility test. The bacterial identification was conducted using Vitek2® (bioMérieux).

\section{Susceptibility Testing}

Susceptibility of bacteria isolates against antimicrobials was detected by Vitek2® (bioMérieux). Susceptibility interpretations were based on CLSI M10o-S23 clinical breakpoints. The antibiotics used included amikacin (AMK), Amoxicillin Clavulanic Acid (AMC), Ampicillin/sulbactam (SAM), aztreonam (ATM), Cefazolin (CZO), cefepime (FEP), cefotaxim (CTX), fosfomycin (FOX), ceftazidim (CAZ), ceftriaxone (CRO), ciprofloxacin (CIP), DOR, doxycycline (DOX), gentamycin (GEN), levofloxacin (LVX), meropenem (MEM), piperacillin/tazobactam (TZP), tigecylin (TGC), tobramycin (TOB), Sulfamethoxazole/ Trimethoprim (SXT) for Gram-negative bacteria. For Gram positive bacteria AMC, SAM, cefadroxil (CFR), FEP, CTX, CAZ, ceftizo- 
xime, (CZX), CRO, cefuroxime (CXM), CIP, carbenicillin (CLI), ertapenem (ETP), erythromycin (ERY), GEN, IPM, LVX, linezolid (LNZ), MEM, tetracyclin (TCY), TGC, SXT, and vancomycin (VAN) were used for analysis.

\section{Data analysis}

Data were analysed in univariate and presented as frequency (percentage).

\section{RESULTS}

There were 1,851 blood positive cultures isolated from patient's blood samples from January 2014- December 2016 in Moewardi Teaching Hospital Surakarta, Indonesia. Various bacteria were isolated; the ten most common bacteria isolated were Klebsiella pneumonia (K. pneumonia), A. baumannii, E. coli, E. cloacae, $P$. aeruginosa, Serratia marcennes (S. mercennes), Staphylococcus haemolyticus (S. haemolyticus), Staphylococcus hominis (S. hominis), Staphylococcus epidermidis (S. epidermidis) and $S$. aureus (see Figure 1). K. pneumoniawas the most common Gram negative bacteria identified (15\%) followed by $A$. baumannii (7\%), E. coli (6\%), E. cloacae (3\%), P. Aeruginosa (3\%), and S. Mercennes (2\%). $S$. haemolyticus was the most common Gram-positive bacteria isolated from blood samples (15\%), followed by $S$. hominis, $S$. epidermidis, and $S$. aureus which accounted for $13 \%, 8 \%$, and $4 \%$, respectively.

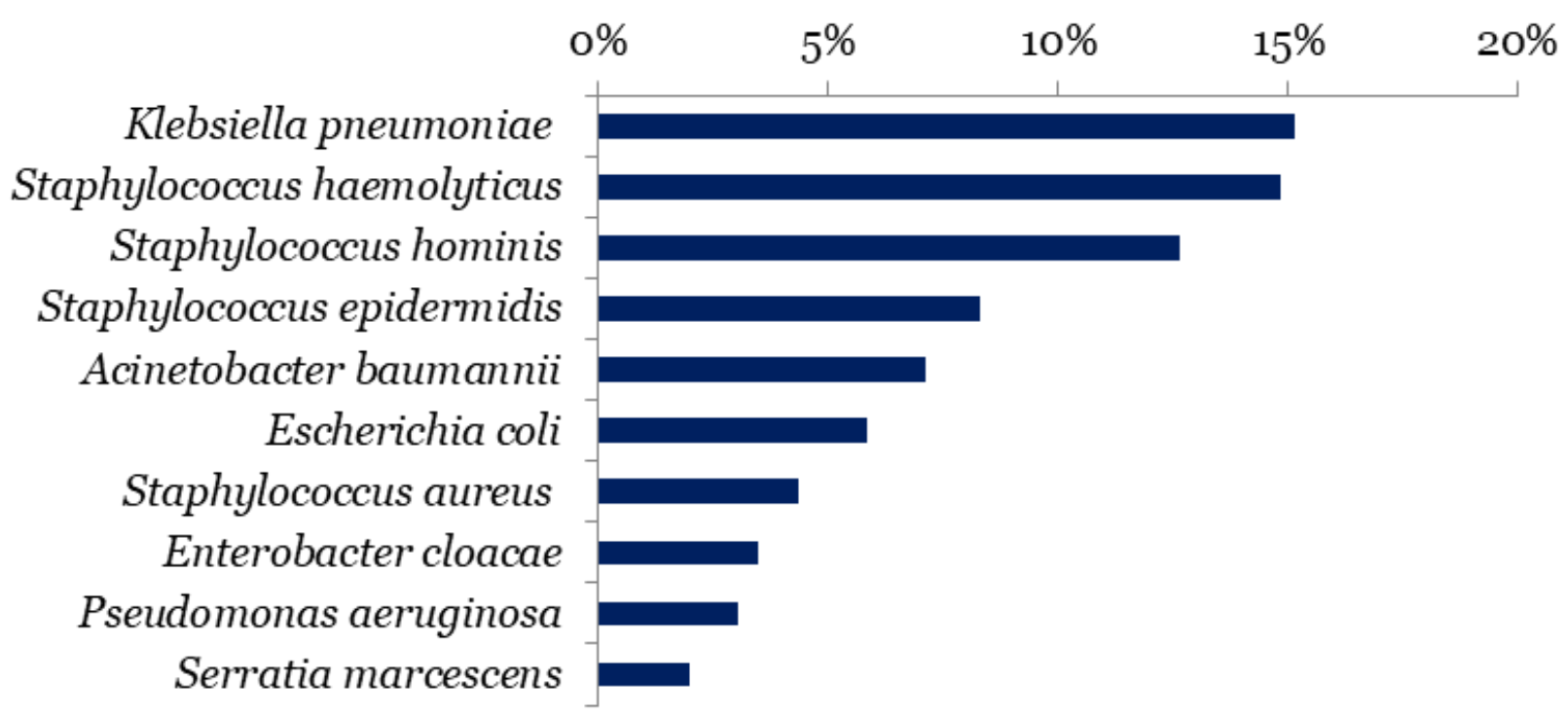

Figure 1. The most common bacteria strains isolated from blood samples of patients with presumptive bloodstream infection during 3 years period (2014 -2016) in Moewardi Teaching Hospital Surakarta

The antibiotic resistant profiles of the most common Gram-negative bacteria isolated from blood samples are shown in Figure 2. The data shows that most of the Gram-negative bacteria isolated were resistant tofirst generation (CZO), second generation (CTX) and third generation cephalosporin (FEP, CRO, CAZ). Out of all Gram-negative bacteria isolated, there were
ESBL-producers. Figure 3 shows the percentage of potential ESBL-producing isolates: $K$. pneumonia (80\%), A. baumannii (92\%), E. coli (77\%), E. cloacae (56\%), P. aeruginosa (92\%), S. marcennes (27\%). The meropnemen resistant bacteria were also detected, such as $P$. aeruginosa (13\%), A. baumannii (41\%), and E. cloacae (4\%). 


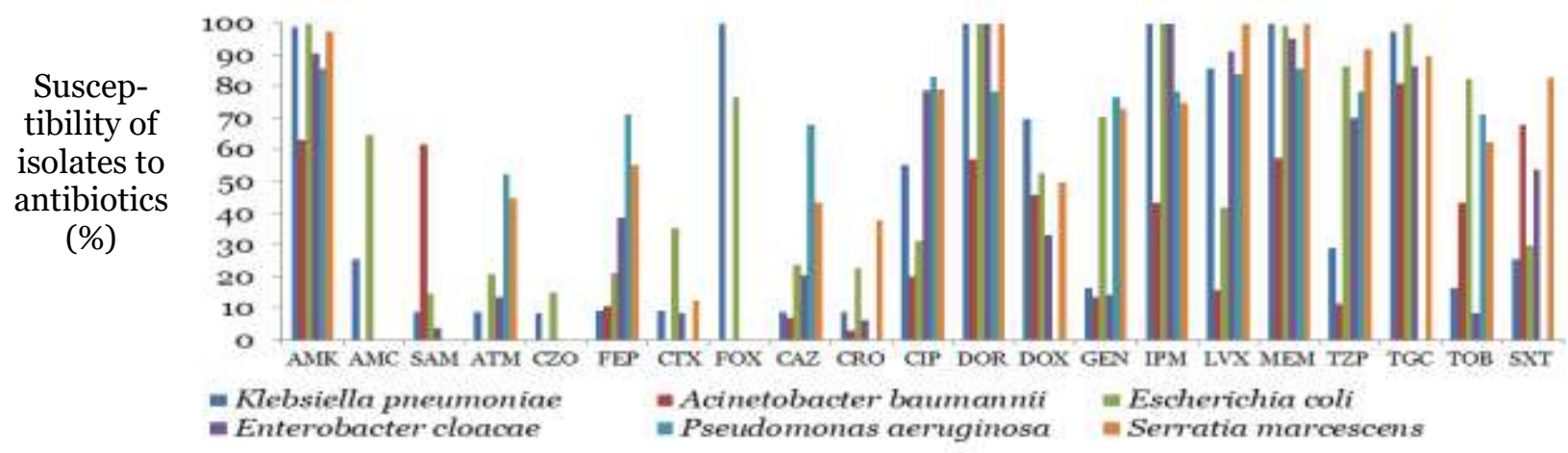

Figure 2. Antibiotic susceptibility profile of Gram negative bacteria isolated from blood samples

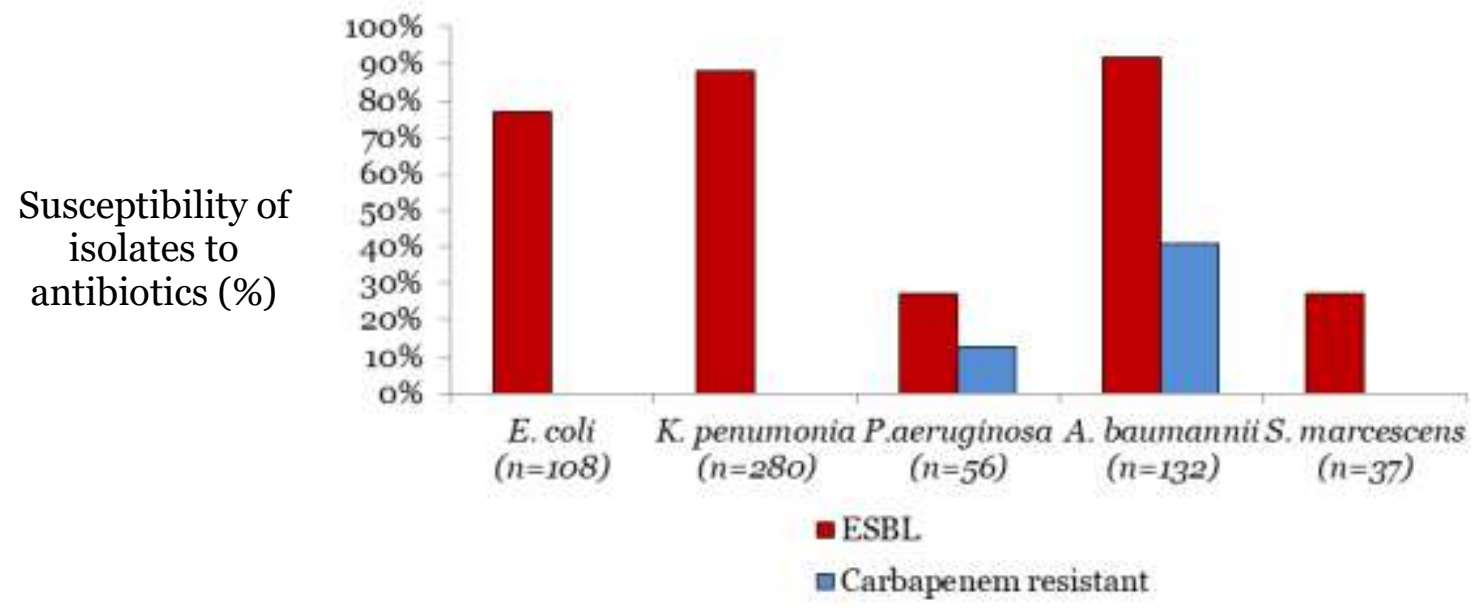

Figure 3. Extended spectrum beta-lactamase-producing bacteria and Carbapenem-resistant strains identified from bacteria isolated from patient's blood samples in three years period

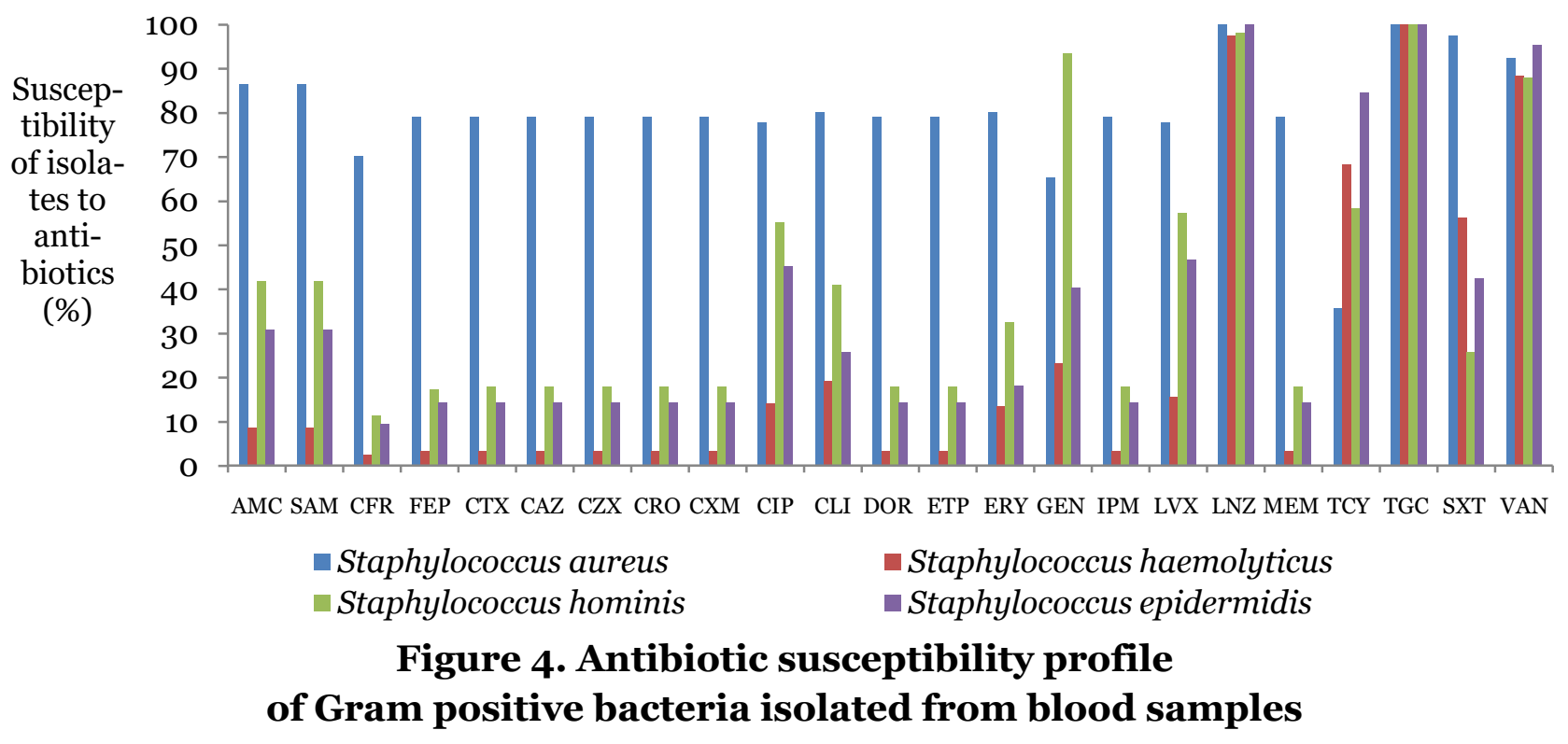


The resistant profile of Gram-positive bacteria showed that the coagulase-negative staphylococci (CoNS) group (S. haemolyticus, S. hominis, and S. epidermidis) were highly resistant to the beta-lactam antibiotics (CFR, FEP, CTX, CAZ, CZX, CRO, CXM), only 5\%-40\%of these strains which still susceptible to these antibiotics (see Figure 3). Out of all the S. aureus strain, $80.5 \%$ of this strain showed susceptibility to these antibiotics and $19.5 \%$ were MDR S. aureus or MRSA (see Figure 4).

\section{DISCUSSION \\ Blood stream infection is conditions asso- ciated with high mortality worldwide and gives a great impact to health care system. It has been previously reviewed that the etiology, antibiotic susceptibility profiles, and the outcome of bloodstream infection were differ between countries and regions ( $\mathrm{Vu}$ Quoc Dat et al., 2017, Buetti et al., 2017). It has been previously showed that MDR bacteria were the most common cause of bloodstream infection in hospital setting, such as Enterococcus sp., Candida spp., Pseudomonas sp., Enterobacter sp., and coagulase-negative staphylococci (Hoenigl et al., 2014). In addition ESBL- producing strain of $E$. coli has been report- ed as a common cause of bloodstream infections in hospitalized patients and in community (Tumbarello et al., 2008).}

The results of this present study showed that MDR bacteria dominantly as the cause of bloodstream infection in the hospital setting. Gram negative bacteria, $K$. pneumonia and Gram-positive bacteria $S$. haemolyticus were the most common agents. Surprisingly, the percentage of Gram-negative bacteria isolated from blood samples showed a multi resistant pattern to various antibiotics tested. Screening of ESBL-producing strains showed that a high number of strains were potential ESBL- producers, such as $K$. pneumonia and $A$. baumannii. This finding is alarming since the increase resistance to drugs included penicillins and cephalosporin has been reported to increase mortality rate (Rodriguez-Bano et al., 2010). In addition, there were a number of $P$. aeruginosa (12.5\%) and $A$. baumannii (40\%) that were resistant to carbapenem drug, meropenem (MEM). Carbapenem drugs were the last resort for MDR bacteria; therefore the finding of meropenem resistant strains is alarming and an effective control and surveillance is required to prevent the spreading of this strain in the hospital environment.

The results also revealed that CoNS group showed multi resistant to various antibiotics, especially beta-lactam antibiotics. The CoNS group is an opportunistic pathogen and can be found as a normal flora in human body. It has been reported that this group was commonly found as the agents causing blood stream infection in hospital setting.

The coagulase positive Staphylococcus, S. aureus, was accounted for the common cause of bloodstream infection. The data showed that $19.5 \%$ of $S$. aureus isolated were MRSA. It has been studied that MRSA bloodstream infection rates were strongly associated with the of antibiotic use (Andreatos et al., 2018). The last resort antibiotic for treatment infection cause by MRSA strain is vancomycin, therefore rational use of this antibiotic has to be carried out in MRSA infection. In addition, it has been suggested that the major cause of the increasing of vancomycin intermediate $S$. aureus and vancomycin resistant $S$. aureus strains (VISA and VRSA respectively) is the irrationals use of broadspectrum antibiotics (Martirosov et al., 2017). Antimicrobial stewardship programs has to be implemented in arresting the 
spread of MRSA (Andreatos et al., 2018) (Duerden et al., 2015), VISA, and VRSA.

The top ten most common bacteria isolated from blood stream were $K$. pneumonia, A. baumannii, E. coli, E. cloacae, $P$. aeruginosa, S. marcennes, $S$. haemolyticus, S. hominis, S. epidermidis, and $S$. aureus. There was a high incidence of MRD bacteria isolated from bloodstream, including ESBL-producing bacteria and MRSA strains. The high prevalence of ESBL-producing bacteria and MRSA in bloodstream infection is alarming, since these bacteria contain mobile drug resistant genes which transferable to other bacteria and therefore can cause an outbreak. Approaches in antibiotic management and bacterial surveillance have to be conducted to reduce the spread of these MDR bacteria.

\section{ACKNOWLEDGMENT}

We thanks to Microbiology laboratory staff at Moewardi Hospital for helping in data access.

\section{REFERENCES}

Andreatos N, Shehadeh F, Pliakos EE, Mylonakis E (2018). The impact of antibiotic prescription rates on the incidence of MRSA bloodstream infections: A county-level, US-wide analysis. Int J Antimicrob Agents, 52: 195-200.

Buetti N, Atkinson A, Marschall J, Kronenberg A, Swiss Centre for Antibiotic R (2017). Incidence of bloodstream infections: a nationwide surveillance of acute care hospitals in Switzerland 2008-2014. BMJ Open, 7: e013665.

Corcione S, Angilletta R, Raviolo S, Filippini C, Fossati L, Di Perri G, Cavallo R, et al. (2018). Epidemiology and risk factors for mortality in bloodstream infection by CP-Kp, ESBL-E, Candida and CDI: A single center retrospective study. Eur J Intern Med, 48: 44-49.

Duerden B, Fry C, Johnson AP, Wilcox MH (2015). The Control of MethicillinResistant Staphylococcus aureus Blood Stream Infections in England. Open Forum Infect Dis, 2: ofvo35.

Hasso M, Porter V, Simor AE (2017). Evaluation of the beta-Lacta Test for Detection of Extended-Spectrumbeta-Lactamase (ESBL)-Producing Organisms Directly from Positive Blood Cultures by Use of Smudge Plates. J Clin Microbiol, 55: 35603562.

Hoenigl M, Wagner J, Raggam RB, Prueller F, Prattes J, Eigl S, Leitner E, et al. (2014). Characteristics of hospitalacquired and community-onset blood stream infections, South-East Austria. PLoS One, 9: e104702.

Kuntaman K, Hadi U, Setiawan F, Koendori EB, Rusli M, Santosaningsih D, Severin J, et al. (2016). Prevalence of Methicillin Resistant Staphylococcus Aureus from Nose and Throat of Patients on Admission to Medical Wards of Dr Soetomo Hospital, Surabaya, Indonesia. Southeast Asian J Trop Med Public Health, 47: 66-70.

Lim SJ, Choi JY, Lee SJ, Cho YJ, Jeong YY, Kim HC, Lee JD, et al. (2014). Intensive care unit-acquired blood stream infections: a 5-year retrospective analysis of a single tertiary care hospital in Korea. Infection, 42: 875-81.

Martirosov DM, Bidell MR, Pai MP, Scheetz MH, Rosenkranz SL, Lodise TP (2017). Relationship between vancomycin exposure and outcomes among patients with MRSA bloodstream infections with vancomycin Etest(R) MIC values of $1.5 \mathrm{mg} / \mathrm{L}$ : A pilot study. Diagn Microbiol Infect Dis, 88: 259263. 
Indonesian Journal of Medicine (2019), 4(1): 28-34

https://doi.org/10.26911/theijmed.2019.04.01.05

Moehario LH, Tjoa E, Rohsiswatmo R, Nursyirwan RS (2012). Microbes profile from blood stream infection cases and their relationship to those of environment in Neonatal unit. Medical Journal of Indonesia.

Pichler G, Pux C, Babeluk R, Hermann B, Stoiser E, De Campo A, Grisold A, et al. (2018). MRSA prevalence rates detected in a tertiary care hospital in Austria and successful treatment of MRSA positive patients applying a decontamination regime with octenidine. Eur J Clin Microbiol Infect Dis, 37: 21-27.

Rodriguez-Bano J, Navarro MD, Romero L, Muniain MA, Cueto M, Galvez J, Perea EJ, et al. (2008). Risk-factors for emerging bloodstream infections caused by extended-spectrum betalactamase-producing Escherichia coli. Clin Microbiol Infect, 14: 180-3.

Rodriguez-Bano J, Picon E, Gijon P, Hernandez JR, Cisneros JM, Pena C, Almela M, et al. (2010). Risk factors and prognosis of nosocomial bloodstream infections caused by extendedspectrum-beta-lactamase-producing
Escherichia coli. J Clin Microbiol, 48: 1726-31.

Tjoa E, Moehario LH, Rukmana A, Rohsiswatmo R (2013). Acinetobacter baumannii: Role in Blood Stream Infection in Neonatal Unit, Dr. Cipto Mangunkusumo Hospital, Jakarta, Indonesia. Int $\mathrm{J}$ Microbiol, 2013: 180763.

Tumbarello M, Sali M, Trecarichi EM, Leone F, Rossi M, Fiori B, De Pascale $\mathrm{G}$, et al. (2008). Bloodstream infections caused by extended-spectrumbeta-lactamase- producing Escherichia coli: risk factors for inadequate initial antimicrobial therapy. Antimicrob Agents Chemother, 52: 324452.

Vu Quoc Dat, Hieu Ngoc Vu, Hung Nguyen The, Hoa Thi Nguyen, Long Bao Hoang, Dung Vu Tien Viet, Chi Linh Bui, et al. (2017). Bacterial bloodstream infections in a tertiary infectious diseases hospital in Northern Vietnam: aetiology, drug resistance, and treatment outcome. BMC Infect Dis. 17: 493. 